\title{
Fever Outpatient Service of General Hospital Applies “1 + 3 + 3" Emergency Management Mode to Deal with COVID-19 Pandemic
}

\author{
Mei Zhang, Wen Yang* \\ The Third Affiliated Hospital of Sun Yat-sen University, Guangzhou, China \\ Email: *154083927@qq.com
}

How to cite this paper: Zhang, M. and Yang, W. (2021) Fever Outpatient Service of General Hospital Applies " $1+3+3$ " Emergency Management Mode to Deal with COVID-19 Pandemic. Open Journal of Emergency Medicine, 9, 115-122. https://doi.org/10.4236/ojem.2021.93011

Received: July 1, 2021

Accepted: August 31, 2021

Published: September 3, 2021

Copyright (c) 2021 by author(s) and Scientific Research Publishing Inc. This work is licensed under the Creative Commons Attribution International License (CC BY 4.0). http://creativecommons.org/licenses/by/4.0/

\begin{abstract}
Purpose: To discuss the effect of " $1+3+3$ " emergency management mode to deal with COVID-19 pandemic in fever outpatient service of general hospitals. Method: This paper analyzes and summarizes the problems encountered by fever outpatient service in dealing with the COVID-19 pandemic from three aspects of "One Team", "Three-Key" Control and "Three Mosts". Results: The application of " $1+3+3$ " emergency management mode can effectively boost the orderliness and efficiency of fever outpatient service in dealing with COVID-19 pandemic. Conclusion: The " $1+3+3$ " emergency management mode provides a new management mode and idea for dealing with COVID-19 pandemic, so the fever outpatient service of general hospitals can better improve the national overall anti-pandemic situation.
\end{abstract}

\section{Keywords}

Fever Outpatient Service of General Hospital, " $1+3+3$ ” Emergency

Management Mode, COVID-19

\section{Introduction}

In recent years, public health emergencies have occurred continuously. While every epidemic disease endangers the health and even lives of the public, it also brings unprecedented challenges to the economy, politics, culture, society, ecology, and national security of the country where it is located. From SAS, H1N1, H7N9, MERS to 2019-nCoV, these public health emergencies have the characteristics of suddenness, severity, frequency, stage and internationality. Not only does it cause serious harm to the people's health and life safety, but it also severely disrupts the national order, which has become a major problem in the 
governance of public health security in my country [1]. As of 17:00 on November 13, 2020, China has recorded 92,386 cases, including 610 existing cases and 3662 cases traveling from abroad. Now there are 14,868,846 confirmed cases overseas. Novel coronavirus pneumonia is mainly characterized by fever, dry cough and fatigue [2]. Therefore, fever outpatient service is deemed as the vanguard in fighting against COVID-19 pandemic and the first protective net to restrain the spread of the virus. By December 2020, more than 7000 fever outpatient services have been set up in general hospitals above the second level in China. As an "outpost" of pandemic, fever outpatient service plays a crucial role in pandemic prevention and control. The first cases of pandemic in Beijing, Dalian and Shanghai were all detected in fever outpatient services [3]. The new type of coronavirus infection pneumonia epidemic spread rapidly and caused serious consequences. As the front line of epidemic prevention and control-fever clinics, how to better respond to public health emergencies and construct a response mechanism for public health emergencies has become a real problem that needs to be solved urgently. The research on emergency management of public health emergencies has also ushered in a new upsurge. Whether the emergency management of fever clinics is scientific, comprehensive, accurate and timely has a direct impact on the development and effectiveness of the hospital's epidemic prevention and control work. Strengthening the emergency management of fever clinics plays an important role in the smooth development of the hospital's epidemic prevention and control work.

\section{2. “1 + 3 + 3” Emergency Management Mode}

The " $1+3+3$ " emergency management model was first proposed by the automobile manufacturing industry, and then gradually applied to hospital management. The " $1+3+3$ " emergency management model can clarify the responsibilities of each functional group, realize a good configuration of emergency response capabilities in emergency situations, optimize the work flow of fever clinic staff to respond to the epidemic, and ensure the efficient and orderly progress of epidemic prevention and control. The fever clinics of the four general hospitals in Guangzhou used the " $1+3+3$ " emergency management model to build a fever clinic emergency system and guarantee system, and built a protective wall for the sniper war against the epidemic. In May 2021, a new round of the 2019-nCoV broke out in Guangzhou, and the first confirmed case was discovered during the surveillance of the fever clinic of a medical institution. The further spread of the 2019-nCoV was prevented in time, and more time was bought for the prevention and control of the 2019-nCoV.

\section{Basic Information}

As one of the first hospitals in Guangdong Province to receive and treat patients with Novel Coronavirus infection, our hospital launched an emergency plan immediately. When the COVID-19 pandemic broke out in December 2019, it 
took us only three days to establish a fever outpatient service promptly. We actively deployed excellent nursing staff from other departments to receive training and take up posts, and set up "three areas and two channels" clearly in strict accordance with the requirements of nosocomial infection, thus achieving orderly treatment for fever patients since the outbreak. From January to December 2020 , a total of 14,062 patients were received to the fever outpatient service of our hospital, including 37 suspected cases, 1 confirmed case and 2 accidents due to mass food poisoning. Neither did cross infection occur among all patients, nor did infection happen among medical staff. The specific measures to fight against COVID-19 by using " $1+3+3$ " emergency management mode in fever outpatient service of our hospital are now reported as follows.

\section{Methods}

\subsection{Basic Content of " $1+3+3$ " Emergency Management Mode}

In the " $1+3+3$ " emergency management mode, " 1 " refers to "one team", that is, an emergency cooperating team led by the chief nurse of fever outpatient service and dominated by nursing staff with strong professional quality and comprehensive ability in each ward of the hospital; The first " 3 " refers to "Three-Key" Control, that is, key training, key symptoms and key areas are taken as the core of emergency control as the pandemic risk changes. The second " 3 " refers to the "Three Most Management", which means to build up the emergency system and guarantee conditions of fever outpatient service based on the principle of "the fastest, most accurate and most effective". The " $1+3+3$ " emergency management mode enables fever outpatient service of general hospitals to respond to public health emergencies quickly and effectively.

\section{2. "One Team"}

Quickly set up a comprehensive medical team consisting of clinicians, nurses, nucleic acid inspectors, transportation personnel, pharmacists, toll collectors, security personnel and cleaning staff. The visiting doctors in fever outpatient service are composed of front-line doctors with rich clinical experience from infectious department, respiratory department, acute and critical illness department, etc., and are strongly backed by a medical treatment team specially set up by the hospital, mainly consisting of specialists and directors in infectious department, infection control department, respiratory department and imaging department. Nursing staff are the key nursing staff in each clinical department, which rotate every 3 - 6 months. Each batch of rotation staff need to work in the department 2 days in advance, so as to be familiar with the department environment, rules and regulations and the latest documents on fever outpatient service issued by the State. All staff must undergo strict assessment and training before they can take up their posts. For nursing staff in fever outpatient service, their age, professional title and specialty background should be comprehensively evaluated in order to achieve resource balance. Attention shall be paid to the 
collocation of the experienced and the young as well as the cooperation and complementarity in specialty to optimize the scheduling mode. The fever outpatient service implements a 24 -hour duty system, and the front-line nurses carry out a "6-4-3" scheduling mode, with 6 shifts for 4 hours each a workday and 3 days off a week, so as to ensure the enough rest of the front-line staff. In addition to the chief nurse, an auxiliary team is set up to cope with emergencies and ensure the supply of front-line protective equipment every day. Each shift is comprised of 2 nurses, 2 doctors, 1 security guard, 1 pharmacist, 1 toll collector, 1 transportation personnel and 1 cleaner to ensure the orderly operation of fever outpatient service [4]. The chief nurse of fever outpatient service should report the daily visits and nucleic acid collection to the medical office every day, and comprehensively register the useful information of fever patients to avoid the mistakes and omissions of patient information.

\section{3. "Three-Key Control"}

The first "3" refers to "Three-Key" Control, that is, key training, key symptoms and key areas are taken as the core of emergency control as the pandemic risk changes.

\subsubsection{Key Training}

Every doctor, nurse and technician working in fever outpatient service must undergo strict training on COVID-19 prevention and control skills. The training mainly includes the process of correctly wearing and removing protective clothing, the compliance and accuracy of hand hygiene, the process and specification of treating items exposed to suspicious contaminants, and the treatment of environment and items [5]. Practice the emergency plan and treatment process of doctors, nurses and technicians with unexpected problems in contaminated areas to ensure their safety. Nursing staff shall master proper methods and cautions of nucleic acid collection, and collect nucleic acid samples expertly; operate the demonstration of emergency equipment and carry out the training of rescuing skills. All staff need to pass the assessment to ensure the medical safety of patients. In addition, they shall treat the specimens of patients with fever correctly to avoid occupational exposure, and formulate emergency plans for occupational exposure.

\subsubsection{Key Symptoms}

Fully implement the pre-examination and triage system and give full play to the "sentinel" role of fever outpatient service as timely detection, rapid disposal, accurate control and effective treatment, so as to work well in the normalization of pandemic prevention and control. Do not miss every patient with fever, cough, fatigue and other symptoms, and examine every patient that should be examined [6]. Take measures such as detection, registration, reporting and guidance. All patients to fever outpatient service must show their "health code" and accept nucleic acid detection. Strictly implement the first diagnosis responsibility sys- 
tem, and do not refuse to accept or treat any patient with fever. If suspicious cases such as fever patients are found, it is necessary to register relevant information in detail and report, treat and transport them in time according to relevant procedures. Try to not miss any patient, report any patient in mistake, or infect any medical staff.

\subsubsection{Key Areas}

Fever outpatient service is set up in an area independent of the inpatient department and outpatient and emergency department of the hospital, which is relatively isolated from the general outpatient department while close to the emergency department. It has independent entrances and exits, which are convenient for screening and transporting patients. The fever outpatient service is provided with separate clinics, waiting area, isolation observation room, pharmacy, registration \& cashier, shelter CT and PCR laboratory. One-stop services, all fever cases are only treated in the fever outpatient service to avoid cross infection. With clear boundaries and striking logo, the "three areas and two channels" includes clean areas, buffer areas, contaminated areas, clean channels and contaminated channels [7]. A password lock is set between the polluted area and the buffer area. Only the staff know the password, preventing patients from entering into the buffer area. Prominent signs can be seen at all entrances, exits and channels to avoid cross-entry and cross-infection.

\section{4. "Three Most" Management}

The second " 3 " refers to the "Three Most Management", which means to build up the emergency system and guarantee conditions of fever outpatient service based on the principle of "the fastest, most accurate and most effective". In view of the normalization of pandemic prevention and control, we should build medical facilities considering "peacetime" with "emergency". The fever outpatient service should be equipped with instruments, facilities and equipment to meet the needs of public health and pandemic prevention, including separate shelter CT. In normal times, all nurses in the hospital take turns at fever outpatient service in batches and departments to ensure each one has skills to response pandemic prevention and control. No matter what happens, each medical staff can work on their posts at any time to battle on the pandemic for adequate personnel reserve. Meanwhile, the hospital established its own PCR laboratory to achieve fast and accurate sampling detection. In normal times, the experimental center undertakes the high-level laboratory testing. However, it also can detect in an emergency, so as to rationally allocate medical resources; improve the management mechanism and management efficiency to give full play to the "energy storage" of the public health system; keep watching the pandemic situation and update strategies of infection prevention and control in time. With the principles of "the fastest, most accurate and most effective", our hospital has established the emergency system and guarantee conditions of the fever outpatient service. 


\section{Implementation of the " $1+3+3$ " Emergency Management Model}

\subsection{Establish a Reform Promotion Team with the Participation of Various Leading Departments}

The implementation of the " $1+3+3$ " emergency management model in hospitals cannot be accomplished by only one department. It requires the joint assistance of various departments such as the nursing department, the material supply department, the logistics office, the medical office, and the infrastructure department. In particular, the leading departments of the hospital need to strengthen the overall deployment of human resources and materials, and supervise the implementation of various measures.

\subsection{Strengthen Implementation and Training}

The ultimate goal of implementing the " $1+3+3$ " emergency management model in hospitals is to improve the governance structure of major epidemic prevention and control, adjust and optimize the decision-making mechanism for epidemic prevention and control, establish a more open epidemic monitoring and response mechanism, and consolidate the organizational guarantees of administrative departments and clinical departments to improve the system and mechanism for responding to public health emergencies. It requires all staff to consciously participate in the " $1+3+3$ " emergency management model. Therefore, in the entire process of implementing the " $1+3+3$ " emergency management model, each department must have an emergency plan for the various tasks of new coronary pneumonia, so as to be able to efficiently and accurately respond to the sudden epidemic. Regularly conduct diversified and professional training for personnel working in key areas, and increase training efforts.

\subsection{Establish an Assessment System}

For fever clinics using the " $1+3+3$ " emergency management model, a prevention and control assessment system has been established to regularly assess key staff. Reward groups or individuals with outstanding performance, increase the enthusiasm of members for work, and appropriately punish groups or individuals with insufficient execution capabilities. Ensure the full implementation of every measure for epidemic prevention and control, and establish a prevention and control system featuring comprehensive mobilization, hospital coverage, full management, full staff assessment, scientific summary, and continuous improvement. Early detection, early reporting, early isolation, early treatment, and comprehensive prevention and control of the novel coronavirus pneumonia epidemic.

Zhou Zhiheng et al. [8] selected 220 community medical institutions from 21 cities in Guangdong Province to conduct an investigation. The results showed that 209 medical institutions had fever clinics, accounting for $95.0 \%$ of the sur- 
veyed institutions in Guangdong Province. Chen Bing et al. [9] investigated the current situation of the establishment, operation and management of fever clinics in medical institutions above the second level in Baoan District, Shenzhen. The results show that the district fever clinic has problems such as insufficient leadership attention, irregular setting, poor operation, insufficient triage execution, unfixed department affiliation and personnel, and inability to develop disciplines and individuals. Fever clinic is one of the key clinics to respond to public health emergencies, and it is also the first line of defense for many medical institutions to prevent infectious diseases. However, the current fever clinics still face operational difficulties such as high-standard construction and cost-benefit games, contradiction between functional positioning and emergency medical needs, and uneven regional development. In order to better play the role of fever clinics in public health emergencies, breakthroughs are needed in their setting and management. We propose to build a " $1+3+3$ " emergency management model for fever clinics in general hospitals, aiming to give full play to the role of infectious disease sentinels in the prevention and medical institutions of fever clinics, and to achieve standardized management of the special department of fever clinics. Provide a basis for my country to improve the emergency management system of public health emergencies.

\section{Response Effects of " $1+3+3$ " Emergency Management in Fighting COVID-19 in the Fever Outpatient Service of Our Hospital}

By the end of December 2020, our hospital has completed the nursing supervision of pandemic prevention and control and nursing service of clinic in four hospital branches. An efficient and ordered stepped team of nursing supervision and clinical care has been reserved. The shift mode is subject to actual adjustment and the supporting staff depend on periodic rotation. The supporting staff works in turn, which ensures continuous supply and reasonable utilization of emergency manpower. Meanwhile, nucleic acid collection points were added to collect nucleic acid for inpatients, companions, overseas personnel, maternal and infant employees and hospital employees. Nucleic acid detection starts for resumption and restart from August 28. The qualified rates of nucleic acid specimens collected, disinfection and isolation measures and outpatient management were $99.98 \%, 98 \%$ and $100 \%$ respectively. A total of 122 orientation trainings were conducted throughout the year, including wearing and taking off protective clothing, disinfection and isolation measures, nucleic acid collection process, suspected cases transferring process, and the use of self-service machines. There is no infection among doctors, nurses and technicians throughout the year, and the training and supervision proceed as normalized COVID-19 prevention and control measures. The fever outpatient service of our hospital was rated as an excellent nursing collective at the hospital level and an advanced collective against COVID-19 pandemic in affiliated universities. 


\section{Conclusion}

In this pandemic prevention and control, the fever outpatient service of our hospital scientifically implements the emergency management of pandemic prevention, forming a multi-disciplinary joint and multi-faceted linkage management mechanism. Various disciplines in the hospital should cooperate and respond in time. The emergency management capacity of fever outpatient services should be improved in all directions, multiple ways and wide coverage. Doctors, nurses and technicians should be taught at all levels to respond scientifically, thus improving the emergency management efficiency.

\section{Conflicts of Interest}

The authors declare no conflicts of interest regarding the publication of this paper.

\section{References}

[1] Zhang, F.T., Li, H. and Wang, L. (2019) " $1+3+3$ " Emergency Management. Modern Occupational Safety, 27-29.

[2] He, L., Bai, Y.X., Lin, D.L., et al. (2020) Study on Ward Management and Infection Control Measures of New Coronavirus Pneumonia. Journal of Xinjiang Medical University, 43, 391-394.

[3] Geng, M.J., Zeng, L.J., Sun, J., et al. (2018) Analysis on Epidemic Situation of Key Infectious Diseases in Winter and Spring of 2017-2018. Disease Surveillance, 33, 179-183.

[4] Yang, F., Yang, Y. and He, L.X. (2021) Application of OEC Mode in Nursing Management of COVID-19 Nucleic Acid Detection Center. Journal of Nursing, 28, 13-16.

[5] Kou, F.Y. and Wang, J.X. (2019) Nosocomial Infection Control Strategy for Measles Outbreak. Gansu Science and Technology, 35, 132-133.

[6] Yu, J.J., Jin, X., Meng, L.L., Wang, X.D. and Liang, Y.N. (2020) Nursing Management Practice of Fever Clinic during COVID-19. Journal of Preventive Medicine of PLA, 38, 112-113.

[7] Yan, Y.Y., Fan, T.Y., Wang, H.T., Zhang, J.M., Du, Z.H. and Sun, X.M. (2021) Standards for Setting up a Fever Sentinel Surveillance Clinic in Primary Healthcare Institutions: Findings of a Qualitative Delphi Study. Chinese General Practice, 24, 1190-1197.

[8] Zhou, Z.H., Wang, C.X., Yu, C.Z., et al. (2014) Assessment of the Current Situation of Related Emergency Clinics and System Setting of Community Medical Institution in Guangdong Province. Chinese General Practice, 2189-2192.

[9] Chen, B., Ma, Z.C., Liu, H., et al. (2016) Setting and Operation of Fever Clinic in Bao'an District of Shenzhen. Chinese Rural Health Service Administration, 36, 207-209. 